\title{
Identifying long indels in exome sequencing data of patients with intellectual disability
}

Sander Pajusalu ${ }^{1,2}$, Rolph Pfundt ${ }^{3}$, Lisenka E.L.M. Vissers ${ }^{3}$, Michael P. Kwint ${ }^{3}$, Tiia

Reimand $^{1,2,4}$, Katrin Õunap ${ }^{1,2}$, Joris A. Veltman ${ }^{3,5}$, Jayne Y. Hehir-Kwa ${ }^{6}$

1. Department of Clinical Genetics, United Laboratories, Tartu University Hospital, Tartu,

Estonia

2. Department of Clinical Genetics, Institute of Clinical Medicine, University of Tartu, Tartu, Estonia

3. Department of Human Genetics, Donders Institute, Radboudumc, Nijmegen, The Netherlands

4. Department of Biomedicine, Institute of Biomedicine and Translational Medicine, University of Tartu, Tartu, Estonia

5. Institute of Genetic Medicine, International Centre for Life, Newcastle University, Newcastle upon Tyne, United Kingdom

6. Princess Máxima Center for Pediatric Oncology, Utrecht, The Netherlands

\section{Corresponding Author:}

Jayne Y. Hehir-Kwa

Princess Máxima Center for Pediatric Oncology

Uppsalalaan 8, 3584CT Utrecht, the Netherlands

E-mail: j.y.hehirkwa@prinsesmaximacentrum.nl 


\begin{abstract}
Exome sequencing is a powerful tool for detecting both single and multiple nucleotide variation genome wide. However long indels, in the size range $20-200 \mathrm{bp}$, remain difficult to accurately detect. By assessing a set of common exonic long indels, we estimate the sensitivity of long indel detection in exome sequencing data to be $92 \%$. To clarify the role of pathogenic long indels in patients with intellectual disability (ID), we analysed exome sequencing data from 820 patients using two variant callers, Pindel and Platypus. We identified three indels explaining the patients' clinical phenotype by disrupting the UBE3A, PGAP3 and $M E C P 2$ genes. Comparison of different tools demonstrated the importance of both correct genotyping and annotation variants. In conclusion, specialized long indel detection can improve diagnostic yield in ID patients.
\end{abstract}




\section{Introduction}

Whole exome sequencing (WES) continues to facilitate the genetic diagnosis of patients with intellectual disability (ID) [1,2]. The sensitivity for identifying single nucleotide variants (SNVs) from next generation sequencing (NGS) data is over 95-99\% [3-5], but is lower when detecting insertions and deletions (indels), particularly in WES data (90-95\%) [4,5]. Indels are known to be a key factor for human genetic disorder pathogenesis due to their damaging effect on protein structure caused by either a translational frame shift or altered protein length [6]. However, due to the difficulties in accurately detecting long indels their contribution to human genetic variation and hereditary disorders is currently under reported in many studies [7]. We aimed to clarify the contribution of pathogenic long indels in patients with ID by using specialized software targeting long indels in WES data.

The majority of SNV and indel detection methods rely on piling up reads, such as Genome Analysis Toolkit's (GATK) UnifiedGenotyper (UG), or local reassembly on regions of interest which is the basis of GATK HaplotypeCaller (HC) [8]. Pile-up based variant callers often fail to detect long indels correctly [9] as they depend heavily on the mapping of sequencing reads and the results of alignment algorithms such as Burrows-Wheeler Aligner (BWA) [10], which apply high penalties for gaps in sequence reads for example involved in large indels. Local reassembly based methods are less dependent on prior mapping of sequence reads for variant calling and as a result have higher sensitivity and specificity in indel calling [11]. Despite their limitations, local reassembly and split-read based methods remain most suited for detecting long indels from WES data. For example, both are challenged by the fragmented nature of WES requiring both breakpoints of deletions to be inside the targeted region. 
We compared two tools for detecting long indels from WES data and applied them to a cohort of 820 ID patients: 1) Pindel, a split-read targeted algorithm using a pattern growth approach [12], and 2) Platypus using local realignment of reads and local assembly [11]. Both tools can detect multiple forms of genetic variation including long indels. Pindel has previously been shown to have higher recall but lower precision rates for indels up to 100bp in size in comparison to GATK UG in WGS data [9]. However unlike Pindel, UG was not able to call indels longer than 100bp [9]. In another study Pindel had higher indel calling sensitivity for WES data if compared to GATK UG and HC [13]. However both studies note the high ratio of false-positive calls for Pindel $[9,13]$. Platypus has been shown to outperform other tools in short indel detection from WGS based data [14]. 


\section{Methods}

Exome sequencing was performed on 820 patient-parent trios with intellectual disability [15]. Briefly, all patients were enrolled in the study from routine clinical diagnostics at the Department of Human Genetics from the Radboud University Medical Center. DNA from the patients was analysed via genomic microarrays for large CNVs as well as exome sequencing for small variants. The exomes were captured using Agilent SureSelect Human All Exon v4 kit and sequenced on an Illumina HiSeq platform with 101-bp paired-end reads to a median coverage of 75×. Mapping was performed by BWA version 0.5.9-r16 [10] and initial variant calling using GATK version 3.4 UG. We then performed variant calling using Pindel version 0.2.5b6 [12] and Platypus version 0.7.9.2 [11]. The variant calling was performed in eight batches of between 102 or 103 samples. Then the variants calls from both tools for all 820 samples were merged together using GATK CombineVariants tool and annotated with Annovar (version date 2015-06-17) [16], SnpEFF (version 4.1) [17] and VEP [18]. We focused our study on indels within size range 20$200 \mathrm{bp}$. The upper limit was selected to match the median length of SureSelect v4 targets to enrich for variants with both breakpoints inside the targeted segments.

To identify clinically relevant long indels, we selected rare disruptive variants, appearing in less than 5\% of the patients overlapping an ID gene list established for diagnostic WES interpretation within our department, version DG 2.3x (http://www.genomediagnosticsnijmegen.nl/services/exome-sequencing-diagnostics). 


\section{Results}

In contrast to SNV, identification the detection and annotation of longer variants such as indels remains challenging. We investigated the contribution of long indels in a cohort of patients 820 with intellectual disability, through the analysis of exome sequencing data.

We first examined the sensitivity of long indel detection of WES by selecting common exonic long indels from ethnically matched whole-genome sequencing (WGS) data derived from The Netherlands (GoNL) dataset [7] and intersecting with the SureSelect v4 targets. This resulted in 25 common (allele frequency (AF) more than 5\%) exonic indels with a size range of 20-200bp and both breakpoints inside the targeted exonic regions. We detected 23 (92\%) of the 25 WGS long indels using Pindel and 9 using Playpus with concordance between AFs of $r=0.94$ (Figure 1). Of the remaining two false negative indels, one occurred in the HLA locus with low mapping quality on chromosome 6 . The second deletion had a breakpoint $9 \mathrm{bp}$ from the end of a capture target, probably causing the failure in detection. UG detected 17 of the 25 variants with an average 10 fold decrease in $\mathrm{AF}$, and failed to detect any indels larger than 50 bp. It should be noted that although most GoNL indels were called using Pindel and Platypus, we observed a trend towards slightly lower AFs for indels identified from exome data (Figure 1). This may suggest some loss in detection sensitivity. In addition, ten more common indels occurred on the border of target capture regions and had only one breakpoint inside the exome target. In this subset, 7 of the 10 indels were identified but with a concordance between AFs of $\mathrm{r}=0.41$. Resulting in a mean decrease of $70 \%$ in AF compared to the WGS AFs. In conclusion, although long indels are rare in exonic regions they can be identified using specialized software tools when both breakpoints are located within the exome capture targets. 
In total 61,052 variants were identified by Pindel and 58,443 by Platypus, of which 3,751 and 1,379 were indels occurring in the size range 20-200bp respectively (Figure 2A). Of which $92 \%$ of variants identified by Pindel and $85 \%$ for Platypus-identified appeared in less than $5 \%$ of individuals (Figure 2A). Variants were further filtered based on quality; variants identified by Pindel requiring, ten or more reads supporting variant (1,396 variants passing) and normalized Phred-scaled likelihood for homozygous-reference genotype of more than 1000 for Platypus-called variants (655 variants passing). After combining variants 1,577 unique long indels were left - 922 called by Pindel, 181 by Platypus and 474 identified by both tools (Figure 2B). 42 indels (33 in-frame, 7 frameshift, stop-gain or splice-site disrupting loss-of-function variants, and two non-exonic variants intersected with the ID gene list (Figure 2C). Many inframe indels were located in repetitive regions, e.g. eight were detected in FMN2 and four in ARID1B.

The seven high-quality loss-of-function variants were further assessed for pathogenicity. Two were heterozygous variants located in recessive disease causing genes SRD5A3 and PRODH. However, no second damaging SNV or CNV could be identified. A 68bp insertion in the exon 10 of $C B S$ gene, was detected in eight patients by Pindel, but is a known benign variant [19]. The remaining four indels represented three separate variants in $P G A P 3$, $U B E 3 A$ and $M E C P 2$ (Table 1). All three variants were classified as pathogenic due to matches with previously reported inheritance patterns and phenotypes in the patients. All three indels were identified in the subset of 325-patients in which no causal variant had previously been identified either via SNV or CNV analysis. Thus, long indels contributed to the diagnosis of these intellectual patients in $0.37 \%$ of cases $(3 / 820)$. 
The first pathogenic indel identified was a 42bp homozygous deletion of exonintron border in PGAP3 gene (NM_033419.3:c.496-39_498del). This deletion was detected by both Platypus and Pindel in a boy with known parental consanguinity, severe ID, epilepsy and microcephaly (Supplementary File 1). Sanger sequencing confirmed the mutation to be heterozygous in both parents and homozygous in proband as well as in an affected sib. This variant was initially detected by UG and determined to be non-pathogenic as it was genotyped as heterozygous and annotated as an inframe deletion. The initial annotation considered only the three deleted exonic nucleotides resulting in a predicted inframe deletion and not predicting the alteration in splice site.

Second, a heterozygous deletion of 36bp segment replaced by $1 \mathrm{bp}$ insertion predicted to cause frameshift in UBE3A gene (NM_130838.1:c.19_54delinsG p.(Lys7Argfs*6)) was called by both Pindel and Platypus (Supplementary File 2). Platypus called the variant as two separate deletions of 6 and 29 bp separated by C-nucleotide matching reference, but Pindel detected the variant as a single indel. Interestingly, the maternal sample showed 5 out of 203 $(2.5 \%)$ reads supporting the same deletion suggesting a mosaic deletion present in the maternal germline. This makes the maternal inheritance highly likely and consistent with Angelman syndrome matching the described patient's phenotype. Subsequent validation with Sanger and Ion Torren PGM sequencing, confirmed the mutation both in the patient and his mother (low mosaic $<10 \%)$.

Finally the third variant consisted of a 114bp heterozygous complex indel disrupting the $M E C P 2$ gene in a girl with severe developmental delay and epilepsy, which was detected by Pindel (NM_004992.3:c.1080_1194delins60 p.(Pro362Glufs*4)) (Supplementary File 3). Both the range of deletion as well as the sequence for inserted sequence described by 
Pindel were confirmed by Sanger sequencing. The mutation appeared de novo, and thus molecular diagnosis could be established due to known X-linked dominant inheritance of MECP2-related disorders such as Rett syndrome.

To further assess the long indel detection capabilities of different variant callers, we performed post hoc analysis using GATK HaplotypeCaller (HC) (version 3.4-46) according to GATK best practice guidelines [20,21] (Table 1.) Both HC and Platypus detected the 36bp and $42 \mathrm{bp}$ deletions, which is expected due to the similar variant calling strategies used for both tools (i.e. local realignment and de novo assembly). Furthermore, both Platypus and HC called the 36bp UBE3A variant as two separate deletions. HC did not detect the $114 \mathrm{bp}$ indel in $M E C P 2$, but 11 de novo heterozygous point mutations and indels up to 24bp in size were called within the genomic segment, thus flagging the region. In contrast to the other variant detection tools, Pindel makes use of discordant read pairs in a pattern growth approach and was able to correctly detect all three pathogenic indels thus outperforming the other variant callers. 


\section{Discussion}

We analysed exome sequencing data derived from 820 patients with intellectual disability and identified 3 clinically relevant long indels which explained the patient's phenotype. However, the application of different variant callers and annotators highlighted both the difficulties and importance of correct genotyping and annotation of long indels. Frequent discordance between annotators is well described, with splice site mutations showing the greatest discrepancy $[22,23]$. This is illustrated by the $42 \mathrm{bp}$ homozygous deletion in PGAP3 which disrupts a splicing site. The variant was called in initial dataset, but was falsely genotyped as heterozygous variant and, moreover, annotated as an inframe exonic deletion. During this study we used two annotators - Annovar (version date 2015-06-17) [16] and SnpEFF (version 4.1) [17]. Additionally, VEP [18] was used post hoc to test the annotation of the PGAP3 deletion. Annovar, also annotated the $42 \mathrm{bp}$ deletion as an in-frame single amino acid deletion if default arguments were used. Both SnpEFF and VEP marked the PGAP3 indel as a high-impact variant and predicted the effect on splicing. Thus, consistent with previous publications we show the crucial role of using suitable variant annotator as well as run parameters for the variant class investigated to enable making correct conclusions on the effect and pathogenicity.

In conclusion, the disruptive indels 20-200bp in size in exons are rare cause for ID, and difficult to accurately detect using standard variant calling software. We have shown, however, that using additional variant callers and annotators can increase the diagnostic yield of WES in patients with ID. 


\section{Acknowledgments}

SP was supported by national scholarship program Kristjan Jaak (Archimedes

Foundation \& Ministry of Education and Research of Estonia) and Liisa Kolumbus scholarship

(The University of Tartu Foundation). The Netherlands Organization for Scientific Research

(NWO) has funded JHK through Veni grant 016.166.015. JV was supported by European

Research Council (ERC) grant DENOVO 281964. KÕ and TR were supported by Estonian

Research Council grant PUT355.

Disclosure statement: The authors declare no conflict of interest. 


\section{References}

1. Vissers LELM, Gilissen C, Veltman JA. Genetic studies in intellectual disability and related disorders. Nat. Rev. Genet. Nature Research; 2015;17:9-18.

2. Deciphering Developmental Disorders Study. Large-scale discovery of novel genetic causes of developmental disorders. Nature [Internet]. 2015;519:223-8. Available from: http://www.ncbi.nlm.nih.gov/pubmed/25533962

3. Meynert AM, Ansari M, FitzPatrick DR, Taylor MS. Variant detection sensitivity and biases in whole genome and exome sequencing. BMC Bioinformatics. BioMed Central; 2014;15:247.

4. Zook JM, Chapman B, Wang J, Mittelman D, Hofmann O, Hide W, et al. Integrating human sequence data sets provides a resource of benchmark SNP and indel genotype calls. Nat. Biotechnol. Nature Research; 2014;32:246-51.

5. Laurie S, Fernandez-Callejo M, Marco-Sola S, Trotta JR, Camps J, Chacón A, et al. From Wet-Lab to Variations: Concordance and Speed of Bioinformatics Pipelines for Whole Genome and Whole Exome Sequencing. Hum. Mutat. 2016;37:1263-71.

6. Mills RE, Pittard WS, Mullaney JM, Farooq U, Creasy TH, Mahurkar AA, et al. Natural genetic variation caused by small insertions and deletions in the human genome. Genome Res. Cold Spring Harbor Laboratory Press; 2011;21:830-9.

7. Hehir-Kwa JY, Marschall T, Kloosterman WP, Francioli LC, Baaijens JA, Dijkstra LJ, et al. A high-quality human reference panel reveals the complexity and distribution of genomic structural variants. Nat. Commun. 2016;7:12989.

8. McKenna A, Hanna M, Banks E, Sivachenko A, Cibulskis K, Kernytsky A, et al. The genome analysis toolkit: A MapReduce framework for analyzing next-generation DNA 
sequencing data. Genome Res. Cold Spring Harbor Laboratory Press; 2010;20:1297-303.

9. Marschall T, Hajirasouliha I, Schönhuth A, Brudno M. MATE-CLEVER:

Mendelian-inheritance-aware discovery and genotyping of midsize and long indels.

Bioinformatics. Oxford University Press; 2013;29:3143-50.

10. Li H, Durbin R. Fast and accurate short read alignment with Burrows-Wheeler transform. Bioinformatics. 2009;25:1754-60.

11. Rimmer A, Phan H, Mathieson I, Iqbal Z, Twigg SRF, Wilkie AOM, et al. Integrating mapping-, assembly- and haplotype-based approaches for calling variants in clinical sequencing applications. Nat. Genet. 2014;46:1-9.

12. Ye K, Schulz MH, Long Q, Apweiler R, Ning Z. Pindel: A pattern growth approach to detect break points of large deletions and medium sized insertions from paired-end short reads. Bioinformatics. Oxford University Press; 2009;25:2865-71.

\section{Ghoneim DH, Myers JR, Tuttle E, Paciorkowski AR. Comparison of} insertion/deletion calling algorithms on human next-generation sequencing data. BMC Res. Notes. BioMed Central; 2014;7:864.

14. Hasan MS, Wu X, Zhang L. Performance evaluation of indel calling tools using real short-read data. Hum. Genomics. BioMed Central; 2015;9:20.

15. Lelieveld SH, Reijnders MRF, Pfundt R, Yntema HG, Kamsteeg E, de Vries P, et al. Meta-analysis of 2,104 trios provides support for 10 novel candidate genes for intellectual disability. Nat. Neurosci. Nature Research; 2016;19:1194-6.

16. Wang K, Li M, Hakonarson H. ANNOVAR: functional annotation of genetic variants from high-throughput sequencing data. Nucleic Acids Res. Oxford University Press; 2010;38:e164. 
17. Cingolani P, Platts A, Wang LL, Coon M, Nguyen T, Wang L, et al. A

program for annotating and predicting the effects of single nucleotide polymorphisms, SnpEff:

SNPs in the genome of Drosophila melanogaster strain w 1118; iso-2; iso-3. Fly (Austin).

2012;6:80-92.

18. McLaren W, Gil L, Hunt SE, Riat HS, Ritchie GRS, Thormann A, et al. The Ensembl Variant Effect Predictor. bioRxiv. BioMed Central; 2016;17:42374.

19. Sperandeo MP, Franchis R, Andria G, Sebastio G. A 68-bp Insertion Found in a Homocystinuric Patient Is a Common Variant and Is Skipped by Alternative Splicing of the Cystathionine $\beta$-Synthase mRNA. Am. J. Hum. Genet. 1966;59:1391-3.

20. DePristo MA, Banks E, Poplin R, Garimella K V, Maguire JR, Hartl C, et al. A framework for variation discovery and genotyping using next-generation DNA sequencing data. Nat. Genet. 2011;43:491-8.

21. Auwera GA Van Der, Carneiro MO, Hartl C, Poplin R, Levy-moonshine A, Jordan T, et al. From FastQ data to high confidence varant calls: the Genonme Analysis Toolkit best practices pipeline. Curr Protoc Bioinforma. 2014;11:1-33.

22. Salgado D, Bellgard MI, Desvignes JP, B??roud C. How to Identify Pathogenic Mutations among All Those Variations: Variant Annotation and Filtration in the Genome Sequencing Era. Hum. Mutat. 2016;37:1272-82.

23. McCarthy DJ, Humburg P, Kanapin A, Rivas M a, Gaulton K, Cazier J-B, et al. Choice of transcripts and software has a large effect on variant annotation. Genome Med. BioMed Central; 2014;6:26. 


\section{Tables}

Table 1. Detected pathogenic long indels and comparison of different variant callers' ability to detect identified pathogenic indels within the study cohort.

\begin{tabular}{|l|l|l|l|l|}
\hline Variant & $\begin{array}{l}\text { Unified } \\
\text { Genotyper } \\
\text { (GATK) }\end{array}$ & Platypus & Pindel & $\begin{array}{l}\text { Haplotype } \\
\text { Caller (GATK) }\end{array}$ \\
\hline $\begin{array}{l}\text { PGAP3: } \\
\text { c.496-39_498del } \\
\text { Homozygous }\end{array}$ & $\begin{array}{l}\text { Called as } \\
\text { heterozygous }\end{array}$ & $\begin{array}{l}\text { Called as } \\
\text { homozygous }\end{array}$ & $\begin{array}{l}\text { Called as } \\
\text { homozygous }\end{array}$ & $\begin{array}{l}\text { Called as } \\
\text { homozygous }\end{array}$ \\
\hline $\begin{array}{l}\text { UBE3A: } \\
\text { c.19_54delinsG } \\
\text { p.(Lys7Argfs*6) } \\
\text { Heterozygous }\end{array}$ & Not called & $\begin{array}{l}\text { Called as two } \\
\text { heterozygous } \\
\text { variants: } \\
\text { c.19_47del and } \\
\text { c.49_54del }\end{array}$ & $\begin{array}{l}\text { Called as } \\
\text { heterozygous }\end{array}$ & $\begin{array}{l}\text { Called as two } \\
\text { heterozygous } \\
\text { variants: } \\
\text { c.19_47del and } \\
\text { c.49_54del }\end{array}$ \\
\hline $\begin{array}{l}\text { MECP2: } \\
\text { c.1080_1194delins60 } \\
\text { p.(Pro362Glufs*4) } \\
\text { Heterozygous }\end{array}$ & Not called & Not called & $\begin{array}{l}\text { Called as } \\
\text { heterozygous }\end{array}$ & Not called* \\
\hline
\end{tabular}

* Haplotype Caller called 11 heterozygous point mutations and indels up to 24bp inside the deleted MECP2 segment.

RefSeq accession numbers: PGAP3 NM_033419.3, UBE3A NM_130838.1, MECP2

NM_004992.3. 


\section{Figures}

Figure 1. The scatter plot comparing allele frequencies for 23 common (allele frequency (AF) above 5\%) indels 20-200 basepairs in size from the Genome of The Netherlands (GoNL) data having both breakpoints inside he SureSelect Human All Exon v4 (Agilent) targets compared to AFs detected from whole exome sequencing of 820 patients with intellectual disabilities. Two exons fulfilling the same criteria which appeared in GoNL data were not called in our ID patient cohort. The concordance $\mathrm{r}=0.94$ is calculated using Pearson method. The linear model based smoothing indicating in blue with standard error in grey. 
Figure 2. Filtering steps used for prioritizing long indels causal for of intellectual disability. A) Number of detected variants per caller after each filtering step. B) Venn diagram showing the intersection between variant callers after filtering high-quality rare 20-200bp indels C) Final filtering steps to identify probably disease-causing variants. 


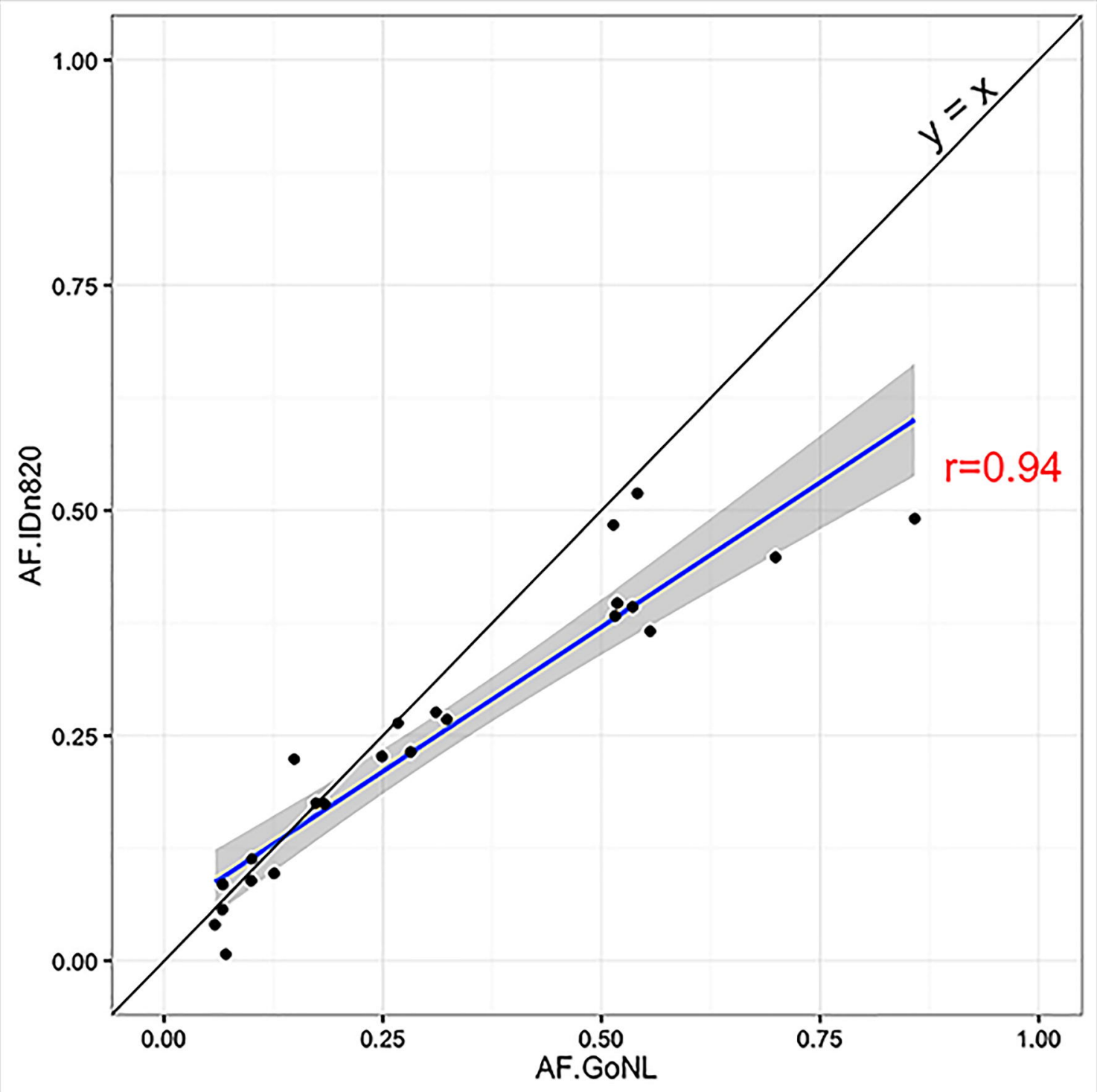




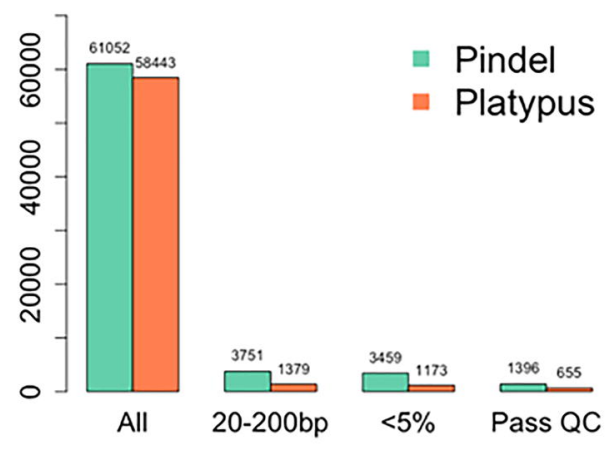

\section{Platypus}

Pindel

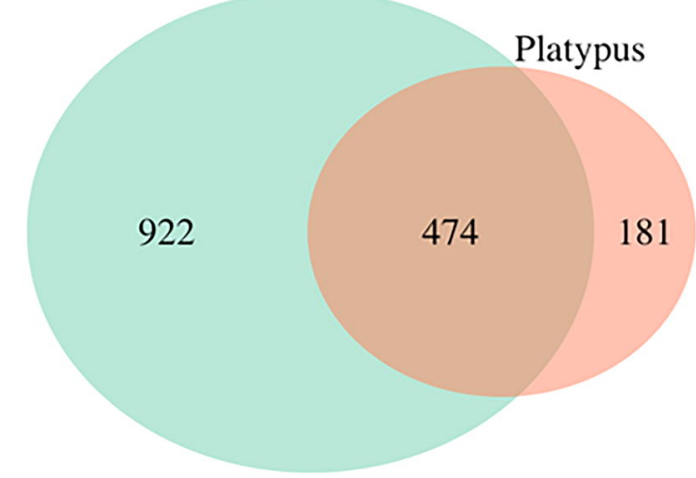

C

1,517

High quality 20-200bp indels

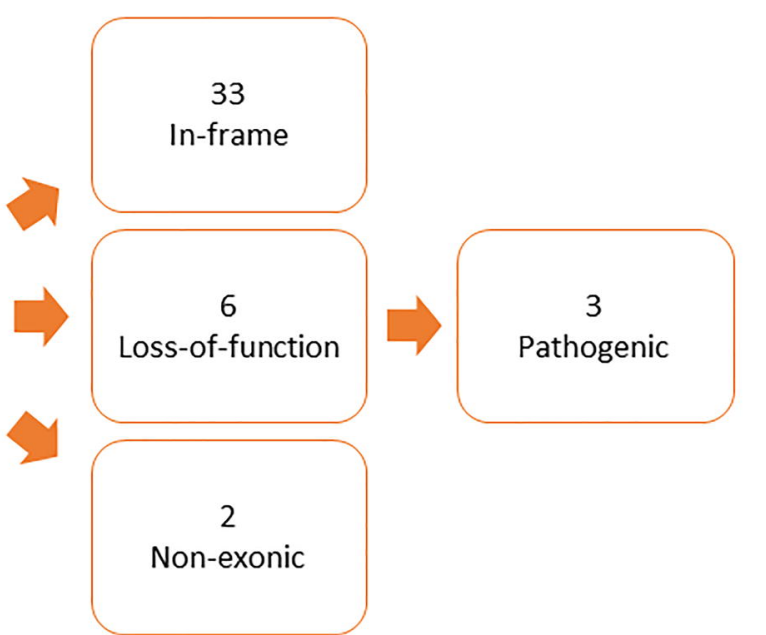

